\section{GREQAM}

Groupement de Recherche en Economie Quantitative d'Aix-Marseille - UMR-CNRS 6579

Ecole des Hautes études en Sciences Sociales Universités d'Aix-Marseille II et III
Document de Travail $n^{\circ}$ 2011-14

\title{
On the optimal control of a linear neutral differential equation arising in economics
}

\author{
Raouf Boucekkine \\ Giorgio Fabbri \\ Patrick A. Pintus
}

March 2011 


\title{
On the optimal control of a linear neutral differential equation arising in economics
}

\author{
R. Boucekkine*, G. Fabbri ${ }^{\dagger} \quad$ P. Pintus ${ }^{\ddagger}$
}

January 21, 2011

\begin{abstract}
In this paper, we apply two optimization methods to solve an optimal control problem of a linear neutral differential equation (NDE) arising in economics. The first one is a variational method, the second follows a dynamic programming approach. Due to the infinite dimensionality of the NDE, the second method requires the reformulation of the latter as an ordinary differential equation in an appropriate abstract space. It is shown that the resulting HJB equation admits a closed-form solution, allowing for a much finer characterization of the optimal dynamics compared to the alternative variational method. The latter is clearly limited by the nontrivial nature of asymptotic analysis of NDEs.
\end{abstract}

Keywords: Neutral differential equations, economic dynamics, optimal control, calculus of variations, dynamic programming, infinite dimension

${ }^{*}$ Corresponding author. IRES and CORE, Université catholique de Louvain, LouvainLa-Neuve, Belgium; GREQAM, France. E-mail: raouf .boucekkine@uclouvain.be

${ }^{\dagger}$ Dipartimento di Studi Economici S. Vinci, Università di Napoli Parthenope, Naples, Italy and IRES, Université catholique de Louvain, Louvain-La-Neuve, Belgium. E-mail: giorgio.fabbri@uniparthenope.it

${ }^{\ddagger}$ GREQAM - Groupement de Recherche en Economie Quantitative d'Aix-Marseille Ecole des Hautes Etudes en Sciences Sociales (EHESS) - CNRS : UMR6579. E-mail: patrick.pintus@univmed.fr 


\section{Introduction}

Delayed systems are increasingly considered in the economic literature. Though their formal introduction in this literature traces back to the 30s (see Kalecki, 1935), they have been seldom studied before the sixties: the vintage capital stream stimulated by Johansen (1959) gave rise to an important mathematical literature on delayed differential (or integro-differential) equations from Solow et al. (1966) to Boucekkine et al. (1997). The optimal control of such systems is treated by much fewer papers in the economic literature: Malcomson (1975), Boucekkine et al. (1997, 2005, 2010), Fabbri and Gozzi (2008) and Fabbri et al. (2008) are among this short list. ${ }^{1}$ Parallel to this literature, and in closer connection with the original Kalecki's paper, some authors have revisited the time-to-build model as an optimal control of delayed differential equations (see Asea and Zak, 1999, Bambi, 2008, Collard et al., 2008, and Bambi et al., 2010). Recently, Bambi and Licandro (2011) have modeled technology diffusion dynamics using delay differential equations by invoking an implementation lag of innovations.

To our knowledge, Boucekkine and Pintus (2010) are the first to identify an economic model leading to a neutral differential equation, that is a functional differential equation where both a variable and its time derivative appear in a retarded form. This type of equations is notoriously much more difficult to handle than the delay differential equations (where the derivatives do not show up in a retarded form). Moreover it is most of the time difficult to extend results obtained on the latter to the former. This is clearly explained in Hale and Verduyn Lunel (1993). The difficulties are numerous. Two are highly sensible: in contrast to delay differential equations, the choice of the initial functional spaces is highly important to produce smooth solutions, non-appropriate choices use to lead to solution paths that are not smoothed with increasing time. More importantly, the usual asymptotic stability theorems relying on the roots of the associated characteristic functions, which work so well on delay differential equations, are potentially misleading in the case of NDEs. Numerous counter-examples can be found in the literature. One of the most famous is due to Brumbey (1970) who showed that an NDE, having all the roots of its characteristic function with strictly negative real part, can nonetheless generate unbounded solutions! Boucekkine and Pintus

\footnotetext{
${ }^{1}$ In the operation research literature, more authors have attempted to tackle the optimal control of vintage capital models: Hritonenko and Yatensko (2005) and Feichtinger et al. (2006) are important contributors to this line of research.
} 
(2010) have exploited a theory developed by Kordonis et al. (1998) for a particular class of NDEs to establish the asymptotic properties of the NDE under consideration.

While the assessment of the asymptotic properties of NDEs is quite challenging, the optimal control of these equations is even more complicated. Accordingly, the related mathematical literature is rather thin, specially when compared to the literature on the optimal control of delay differential equations. It is well known that the maximum principle is more difficult to formulate and to apply in the case of the NDEs (see Kolmanovski and Myshkis, 1999, chapter 14). Indeed, the most important control literature related to the NDEs concerns robust control of uncertain linear NDEs (for example, see Xu et al., 2002). The objective of this paper is to address in detail the optimal control of the linear NDE identified in the economic model by Boucekkine and Pintus (2010). Incidentally, a methodological discussion will be conducted with the aim to identify the main advantages of variational methods compared to dynamic programming.

Actually, we shall try to replicate two techniques already applied for the optimization of linear delay differential equations in the economic literature. One is the variational method applied by Boucekkine et al. (2005) to solve the socalled vintage capital AK model, and the other is the dynamic programming method implemented by Fabbri and Gozzi (2008) to solve the same model. ${ }^{2}$ Because the NDEs (just like delay differential equations) are infinitely dimensioned, the application of dynamic programming requires a non-trivial adaptation work: this is usually done by reformulating the functional differential equations as ordinary differential equations in properly defined abstract Hilbert spaces, thus resulting in formally standard HJB equations (see a general presentation in Bensoussan et al., 2007). Fabbri and Gozzi (2008) applied successfully this technique to solve the vintage AK model which gives rise to a linear delay differential equation. Moreover, they found a closedform solution to the value function of the problem, and thus to its feedback, which allowed them to reach a finer characterization of the optimal dynamics compared to Boucekkine et al. (2005).

In this paper, we shall argue that the comparative advantage of dynamic programming is even larger when dealing with linear NDEs. Indeed, we will show that it is still possible to reformulate them as evolution equations (a sort of ODEs) in appropriate abstract spaces using the work of Burns et al.

\footnotetext{
${ }^{2}$ See also Fabbri et al.(2008).
} 
(1983) and Kappel and Zhang (1986), and to identify a closed-form solution to the corresponding HJB equation, resulting in a much finer characterization of the optimal dynamics compared to the alternative variational method. The latter is clearly limited by the nontrivial nature of asymptotic analysis of linear NDEs.

This paper is organized as follows. Section 2 describes briefly the model and states the corresponding optimal control problem. Section 3 highlights the outcomes of the variational method. Section 4 gives the outcomes of the dynamic programming approach. Section 5 develops the mathematical steps taken in the application of the latter approach, and proves the main related claims in Section 4.

\section{The problem}

The problem is an optimal control version of the model developed in Boucekkine and Pintus (2010). ${ }^{3}$ It comes from the modelling of an open economy which borrows in international markets at given interest rate $r$ in order to finance capital accumulation. The main departure from standard theory is that due to imperfect observability, lenders can only rely on lagged information when evaluating the amount to be lent, which is quite realistic. More precisely, the model aims at capturing borrowing without precommitment in investment: it entails a credit ceiling equal to $\lambda K(t-\tau)$, for some exogenous informational delay $\tau \geq 0$ and $\lambda>0$. If credit constraint is binding, then $D(t)=\lambda K(t-\tau)$ relates borrowing to collateral.

The economy produces a tradeable good $Y$ by using physical capital $K$, according to the following technology:

$$
Y=A K,
$$

where $A>0$ is total factor productivity. Output is tradeable, whereas labor and capital are not. Households have to maximize their intertemporal utility:

$$
\int_{0}^{\infty} e^{-\rho t} \frac{C(t)^{1-\sigma}-1}{1-\sigma} d t
$$

where $C>0$ is consumption, $\sigma \geq 0$, and $\rho \geq 0$ is the discount rate. It is worth mentioning that we are considering a standard utility function, called

\footnotetext{
${ }^{3}$ This problem is stated in the appendix of the latter paper but it is only partially addressed.
} 
iso-elastic utility function, which has the virtue to be homogenous in the control. The budget constraint is:

$$
\dot{K}(t)-\dot{D}(t)=A K(t)-\delta K(t)-r D(t)-C(t),
$$

where $D$ is the amount of net foreign debt and the initial stocks $K(0)>0$, $D(0)$ are given to the households. Replacing $D$ by its expression given above, budget constraint (3) can be written as:

$$
\dot{K}(t)=\lambda \dot{K}(t-\tau)+(A-\delta) K(t)-r \lambda K(t-\tau)-C(t),
$$

We consider the problem of maximizing the intertemporal welfare function (2) under the budget constraint (4) and appropriate boundary conditions. There are two major difficulties in the latter state equation. First, the state variable, $K($.$) , shows up in a lagged form: we therefore face a func-$ tional differential equation, which induce an infinitely dimensioned problem. Problems with lagged state or control variables in the state equation are already considered in the economic literature: see for example Boucekkine et al. (2010), Bambi (2008), Fabbri and Gozzi (2008), Boucekkine et al. (2005) or Asea and Zak (1999). More importantly, the time derivative of the state variable also shows up in a lagged form, (4) is called a neutral differential equation. Boucekkine and Pintus (2010) has solved the Solow version of the model, that is under the assumption of constant saving rate, $s$, giving rise to the scalar linear neutral differential equation:

$$
\dot{K}(t)=\lambda \dot{K}(t-\tau)+(s A-\delta) K(t)-r \lambda K(t-\tau) .
$$

Boucekkine and Pintus (2010) have studied the asymptotic properties of the solutions to the latter equation relying on previous work of Kordonis et al. (1998). In particular, they have showed that there exists a balanced growth solution to the equation (that's a an exponential solution with positive exponent) and that this trajectory is asymptotically stable (see Proposition 2.4). To our knowledge, this work is the first one in the economic literature dealing with neutral differential equations. In this paper, we aim to handle the full problem, that is the initial optimal control version of it. More precisely, we develop two methods to tackle the optimal control problem. One is inspired from Boucekkine et al. (2005): it simply tries to adapt the usual variational approach to the control of delayed differential equations. The second one is the dynamic programming approach advocated by Bensoussan et al. (2007). 
It will be argued that the latter approach is the most efficient in handling the type of problems considered in this paper, that is problems where the objective function is homogenous in the control and the state equation is a linear neutral differential equation (NDE). As argued in the introduction, the advantage of the dynamic programming method comes from the fact it permits to identify a closed-form solution for the value function and the feedback while only asymptotic results are possible with the standard variational method due to the complexity of neutral equations.

\section{The variational approach}

In this section, we replicate the simple variational approach implemented by Boucekkine et al. (2005) for the optimal control of a delayed differential equation. We shall stick here to the traditional definition of optimal controls. ${ }^{4}$

Definition 3.1. Given an initial profile for capital, $K_{I}(t)$, on $[-\tau, 0]$, where $K_{I}(t)$ is piecewise differentiable, a trajectory $(C(t), K(t)), t \geq 0$, is admissible if it checks (4) with $C(t)$ positive and piecewise continuous, $K(t)$ positive and piecewise differentiable, and such that the integral (2) is convergent. A trajectory $\left(C^{*}(t), K^{*}(t)\right)$ is optimal if it is admissible and if for any admissible trajectory $(C(t), K(t))$, the value of $(2)$ is not greater than its value under $\left(C^{*}(t), K^{*}(t)\right)$.

The definition is standard except that we require that an initial piecewise differentiable function $K_{I}(t)$, on $[-\tau, 0]$ is given. These data are needed for the solution to delayed differential equations to be well defined. We now focus on the derivation of first-order optimality conditions for interior maximizers using calculus of variations. The corresponding proposition is given here ${ }^{5}$ :

Proposition 3.2. If $(C(t), K(t)), t \geq 0$, is an interior optimal solution, then there exists a piecewise differentiable equation $q(t)$ such that for all $t \geq 0$ :

$$
q(t)=e^{-\rho t} C(t)^{-\sigma}
$$

\footnotetext{
${ }^{4}$ Additional considerations should be in principle addressed when defining a maximum principle in presence of NDEs, see Kolmanovski and Myshkis (1999), Section 5.5, chapter 14. We abstract away from them here, our unique aim being the identification of the deepest characterization of optimal dynamics allowed by the variational approach.

${ }^{5}$ This proposition is given without proof in the appendix of Boucekkine and Pintus (2010), we develop here the variational argument.
} 


$$
(A-\delta) q(t)+\dot{q}(t)-r \lambda q(t+\tau)-\lambda \dot{q}(t+\tau)=0
$$

Proof. : We start by denoting $V(T)$, with $T>\tau$, the value of the finite time horizon integral: $V(T)=\int_{0}^{T} e^{-\rho t} \frac{C(t)^{1-\sigma}-1}{1-\sigma} d t$. If $q(t)$ is the co-state variable associated with the state equation (4), one can write:

$$
\begin{aligned}
V(T)=\int_{0}^{T} e^{-\rho t} \frac{C(t)^{1-\sigma}-1}{1-\sigma}+q(t)(\lambda \dot{K}(t-\tau) \\
\quad+(A-\delta) K(t)-r \lambda K(t-\tau)-C(t)-\dot{K}(t)) d t .
\end{aligned}
$$

Now integrate by part $q(t)(\lambda \dot{K}(t-\tau)-\dot{K}(t))$ :

$$
\begin{aligned}
\int_{0}^{T} q(t)( & \lambda \dot{K}(t-\tau)-\dot{K}(t)) d t=q(T)(\lambda K(T-\tau)-K(T)) \\
& +q(0)(K(0)-\lambda K(-\tau))-\int_{0}^{T} \dot{q}(t)(\lambda K(t-\tau)-K(t)) d t
\end{aligned}
$$

which implies that:

$$
\begin{aligned}
& V(T)=\int_{0}^{T} e^{-\rho t} \frac{C(t)^{1-\sigma}-1}{1-\sigma}+q(t) \\
&\quad-r \lambda K(t-\tau)-C(t))-\dot{q}(t)(\lambda K(t-\tau)-K(t)) d t \\
& \quad+q(T)(\lambda K(T-\tau)-K(T))+q(0)(K(0)-\lambda K(-\tau)) .
\end{aligned}
$$

Let us take now the first variation of $V(T)$ : we get

$$
\begin{aligned}
\delta V(T)=\int_{0}^{T}\left\{\left(e^{-\rho t} C(t)^{-\sigma}-q(t)\right) \delta C(t)\right. & \\
+((A-\delta) q(t)+\dot{q}(t)) \delta K(t) & +(-r \lambda q(t)-\lambda \dot{q}(t)) \delta K(t-\tau)\} d t \\
& +\lambda q(T) \delta K(T-\tau)-q(T) \delta K(T) .
\end{aligned}
$$


Non-standard terms $\delta K(t-\tau)$ show up. However, notice that:

$$
\begin{aligned}
& \int_{0}^{T}(-r \lambda q(t)-\lambda \dot{q}(t)) \delta K(t-\tau) d t \\
&=\int_{-\tau}^{T-\tau}(-r \lambda q(t+\tau)-\lambda \dot{q}(t+\tau)) \delta K(t) d t
\end{aligned}
$$

which implies since $K(t)=K_{I}(t)$ given on $[-\tau, 0]$ :

$$
\begin{aligned}
& \int_{0}^{T}(-r \lambda q(t)-\lambda \dot{q}(t)) \delta K(t-\tau) d t= \\
& \int_{0}^{T-\tau}(-r \lambda q(t+\tau)-\lambda \dot{q}(t+\tau)) \delta K(t) d t .
\end{aligned}
$$

Substituting in $\delta V(T)$, one can then extract the first-order conditions in the traditional way, that is by checking that $0 \geq \delta V(T)$ for all admissible perturbation $(\delta C(t), \delta K(t))$ for $t \in[0, T]$. In particular, one gets the usual optimality condition with respect to consumption:

$$
e^{-\rho t} C(t)^{-\sigma}-q(t)=0 \text {. }
$$

With respect to the state variable, things are much more involved. On the interval $[0, T-\tau]$, it is:

$$
(A-\delta) q(t)+\dot{q}(t)-r \lambda q(t+\tau)-\lambda \dot{q}(t+\tau)=0,
$$

while on the interval $[T-\tau, T]$, it simplifies into the ODE

$$
(A-\delta) q(t)+\dot{q}(t)=0 .
$$

Unfortunately, when $T$ tends to infinity, the relevant equation turns out to be the first one. This ends the proof.

It's then easy to study the asymptotic properties of the neutral advanced equation (7). As argued in Boucekkine and Pintus (2010), the asymptotic properties of the latter are symmetrical to those of the neutral delayed equation (5), with $s=1$. More precisely, while equation (5) admits two balanced growth paths, one with positive growth rate and the other with negative growth rate, only the former being asymptotically stable (Proposition 2.4 in 
Boucekkine and Pintus, 2010), the advanced equation (7) has the symmetrical property: only the balanced growth path for $q(t)$ with negative growth rate is asymptotically stable. ${ }^{6}$ Because of the Euler equation (6), this implies that the balanced growth path with growing consumption (and capital) at positive rate is asymptotically stable, which is a natural outcome since the production function is AK. As mentioned in the introduction, the main mathematical argument to establish these asymptotic stability properties is extracted from Kordonis et al. (1998), which also provide conditions for non-oscillatory solution paths. In short, the asymptotic analysis of (6)-(7) permits to conclude that consumption and capital will behave asymptotically as exponential functions at a well identified growth rate. We have not been able to push this argument any further with the tools of asymptotic analysis of linear NDEs. We show hereafter that the dynamic programming approach, already implemented by Fabbri and Gozzi (2008) in the delayed case, provides a much finer characterization of the asymptotics: more precisely, it will be established that optimal consumption is actually constant once detrended (that is when exponential growth at the rate of the balanced growth path is removed). In other words, the dynamic programming method allows to prove that optimal consumption is exponential from $t=0$ while the variational approach only permits to conclude that consumption is exponential asymptotically. The superiority of this method derives, as we will show, from the identification of closed-form solutions to the corresponding HJB equations, which is not that surprising given the linearity of the state equation.

\section{The dynamic programming approach}

In order to ease the presentation, we shall proceed in two steps. In the first step, we give the main outcomes of the dynamic programming approach in order to compare immediately with those of the variational method. In particular, the constancy of optimal detrended consumption will be put forward. This is the objective of this section. In the next section, we will develop the dynamic programming approach including the prior work required to transform the NDE into an evolution equation in an appropriate abstract space.

\footnotetext{
${ }^{6}$ The symmetry is a direct consequence of the fact that the characteristic function of (7) can be obtained from the one of (5) by an elementary linear transformation.
} 
We start by an estimate of maximal growth of the solutions to the NDE, which is a necessary step to establish conditions under which the value function is finite. Consider the neutral differential equation

$$
\left\{\begin{array}{l}
\dot{K}(t)=\lambda \dot{K}(t-\tau)+(A-\delta) K(t)-r \lambda K(t-\tau)-C(t) \\
K(s)=K_{I}(s) \quad \text { for all } s \in[-\tau, 0] .
\end{array}\right.
$$

where $\tau, A, \delta, r$ are positive constant and $K_{I}:[-\tau, 0] \rightarrow \mathbb{R}$ is a continuous function. Given a control $C(\cdot) \in L_{l o c}^{2}(0,+\infty)$ we say that $K \in C([-\tau,+\infty))$ is a solution of (14) if $K(s)=K_{I}(s)$ for all $s \in[-\tau, 0]$ and, for all $t \geq 0$, one has

$$
\begin{aligned}
K(t)-\lambda K(t-\tau)=K(0)-\lambda K(-\tau) & +\int_{0}^{t}(A-\delta) K(s) \mathrm{d} s \\
& -\int_{0}^{t} \tau r K(s-\tau) \mathrm{d} s-\int_{0}^{t} C(s) \mathrm{d} s .
\end{aligned}
$$

Given a control $C(\cdot) \in L_{\text {loc }}^{2}(0,+\infty)$ (14) has a unique continuous solution $K_{C}(\cdot)$ on $[-\tau,+\infty)$ (see Hale and Verduyn Lunel (1993) Theorem 1.1 page 256).

Consider the uncontrolled equation

$$
\dot{K}(t)=\lambda \dot{K}(t-\tau)+(A-\delta) K(t)-r \lambda K(t-\tau),
$$

its characteristic equation is given (see Hale and Verduyn Lunel (1993) page 264, equation (2.8)) by

$$
z-\lambda z e^{-z \tau}-(A-\delta)+r \lambda e^{-z \tau}=0 .
$$

Proposition 4.1. Assume that $A, \delta, r \geq 0$,

$$
A-\delta-r>0
$$

and that

$$
\lambda \leq 1
$$

then the characteristic equation (16) has a unique (real and) positive root $\xi$.

Proof. Call $T(z):=z-\lambda z e^{-z \tau}-(A-\delta)+r \lambda e^{-z \tau}$ (for real and positive $z$ ). We have that

$$
\lim _{z \rightarrow 0^{+}} T(z)=-(A-\delta-\lambda r)<0
$$


and

$$
\lim _{z \rightarrow+\infty} T(z)=+\infty
$$

so at least a positive root exists. Let us compute

$$
\frac{\mathrm{d}}{\mathrm{d} z} T(z)=1-\lambda e^{-z \tau}+\lambda \tau z e^{-z \tau}-r \lambda \tau e^{-z \tau} .
$$

Let us compute such an expression at a certain root $\bar{z}$ of the characteristic equation, from (16) we have that, $\lambda \tau \bar{z} e^{-\bar{z} \tau}-r \lambda \tau e^{-\bar{z} \tau}=\tau(\bar{z}-(A-\delta))$ so the derivative of $T$ at $\bar{z}$ is

$$
\frac{\mathrm{d}}{\mathrm{d} z} T(\bar{z})=F(\bar{z}):=1-\lambda e^{-z \tau}+\tau(\bar{z}-(A-\delta)) .
$$

$F$ Is strictly increasing in $\bar{z}$, so if $\bar{z}_{1}<\bar{z}_{1}$ are two positive roots one has $\frac{\mathrm{d}}{\mathrm{d} z} T\left(\bar{z}_{1}\right)<\frac{\mathrm{d}}{\mathrm{d} z} T\left(\bar{z}_{2}\right)$. Let us call $\xi$ the smallest (strictly) positive root. Since $T(0)<0$ then $\frac{\mathrm{d}}{\mathrm{d} z} T(\xi) \geq 0$ and so $\frac{\mathrm{d}}{\mathrm{d} z} T(\bar{z})>0$ for all the roots greater than $\xi$. This means that $\xi$ is indeed the only positive root. This concludes the proof.

We want to study the problem of maximizing

$$
\int_{0}^{+\infty} e^{-\rho t} \frac{C^{1-\sigma}(t)}{1-\sigma} \mathrm{d} t
$$

subject to (14), where $\rho$ and $\sigma$ are fixed strictly positive constant ( $\sigma$ not equal to 1) among the set

$$
\mathcal{U}:=\left\{C:[0,+\infty): C \in L_{l o c}^{2}(0,+\infty), c \geq 0, K_{C} \geq 0\right\} .
$$

The value function of the problem is defined as:

$$
V\left(K_{I}\right):=\sup _{C \in \mathcal{U}} \int_{0}^{+\infty} e^{-\rho t} \frac{C^{1-\sigma}(t)}{1-\sigma} \mathrm{d} t .
$$

Lemma 4.2. Assume that hypotheses of Proposition 4.1 are satisfied. Call $K_{M}(\cdot)$ the unique solution of (14) when one choose $C \equiv 0$. Then for every choice of $C \in \mathcal{U}$ we have $K_{C}(t) \leq K_{M}(t)$ for all $t \geq 0$. Moreover, for every $\varepsilon>0$ one has

$$
\lim _{t \rightarrow+\infty} K_{M}(y) e^{-t(\xi+\varepsilon)}=0
$$


Proof. The first statement follows easily by the definition of solution. The second one can be found for example in Hale and Verduyn Lunel (1993) Corollary 3.1 page 274 .

After this preliminary analysis, it is now possible to identify the conditions under which the value function is finite, and to examine the solution to the HJB equation (see next section for details on the construction of the HJB equation in this context. Even more, it is possible to find an explicit solution to this equations, which will ease the characterization of optimal control paths. This is done in the next propositions:

Proposition 4.3. Assume that hypotheses of Proposition 4.1 hold. Moreover assume that

$$
\rho-(1-\sigma) \xi>0
$$

then

$$
V\left(K_{I}\right)<+\infty \text {. }
$$

Proof. It is a corollary of Lemma 4.2 .

Theorem 4.4. Assume that hypotheses of Proposition 4.1 are satisfied. Moreover assume that (23) is satisfied and define

$$
\gamma:=\left(\frac{\rho-\xi(1-\sigma)}{\sigma}\right)
$$

Then, provided that the related trajectory $K^{*}(t)$ remains positive, the optimal control of the system can be expressed in feedback form as

$$
\Phi(K):=\gamma\left[(K(0)-\lambda K(-\tau))+(\delta+\xi-A) \int_{-\tau}^{0} e^{-\xi s} K(s) \mathrm{d} s\right]
$$

So the optimal trajectory is given by the unique solution $K^{*}(t)$ of

$$
\left\{\begin{aligned}
\dot{K}^{*}(t) & =\lambda \dot{K}^{*}(t-\tau)+(A-\delta) K^{*}(t)-r \lambda K^{*}(t-\tau) \\
-\gamma & {\left[(K(t)-\lambda K(t-\tau))+(\delta+\xi-A) \int_{-\tau}^{0} e^{-\xi s} K(t+s) \mathrm{d} s\right] } \\
K^{*}(s) & =K_{I}(s) \quad \text { for all } s \in[-\tau, 0],
\end{aligned}\right.
$$


the optimal control is given by $C^{*}(t)=\Phi\left(K^{*}(t)\right)$ and the value function can be written explicitly as

$$
V\left(K_{I}\right)=\alpha\left[\left(K_{I}(0)-\lambda K_{I}(-\tau)\right)+(\delta+\xi-A) \int_{-\tau}^{0} e^{-\xi s} K_{I}(s) \mathrm{d} s\right]^{1-\sigma}
$$

where

$$
\alpha=\frac{1}{1-\sigma}\left(\frac{\rho-\xi(1-\sigma)}{\sigma}\right)^{-\sigma} .
$$

Proposition 4.5. Assume that hypotheses of Theorem 4.4 are satisfied. Then the optimal control $C^{*}(t)$ of the system is

$$
C^{*}(t):=C_{0} e^{g t}
$$

where

$$
g:=\frac{\xi-\rho}{\sigma}
$$

and

$$
C_{0}:=\gamma\left[\left(K_{I}(0)-\lambda K_{I}(-\tau)\right)+(\delta+\xi-A) \int_{-\tau}^{0} e^{-\xi s} K_{I}(s) \mathrm{d} s\right] .
$$

Theorem 4.4 and Proposition 4.5 contain the main results of the section: on one hand the explicit optimal feedback rule (the "policy function") of the problem (14)-(21)-(22) given in (25) and the explicit form of the value function (given in (27)), on the other hand, in (29), a complete description of the optimal control and the NDE (26) that describes the evolution of the capital stock along the optimal path. Detailed proofs of Theorem 4.4 and Proposition 4.5 are given in the next section (in particular in Subsection 5.2). At the minute, notice that as announced before, detrended optimal consumption is constant, a property which also prevails in standard AK theory. It is possible to fully study capital dynamics. As it is shown below, detrended capital is not constant in general but it converges, as established by the variational approach above, to an exponential path with the same growth rate as consumption.

The following corollary gives another form for the NDE that describes the evolution of the optimal capital: 
Corollary 4.6. Assume that hypotheses of Theorem 4.4 are satisfied. Then the optimal trajectory of the capital driven by the optimal control is the unique solution of

$$
\left\{\begin{array}{l}
\dot{K}^{*}(t)=\lambda \dot{K}^{*}(t-\tau)+(A-\delta) K^{*}(t)-r \lambda K^{*}(t-\tau)-C_{0} e^{g t} \\
K^{*}(s)=K_{I}(s) \quad \text { for all } s \in[-\tau, 0] .
\end{array}\right.
$$

Proof. The expression follows from (14) once we substitute the expression for the optimal consumption given in (29).

For notational convenience we introduce

$$
\left\{\begin{array}{l}
R: C([-\tau, 0]) \rightarrow \mathbb{R} \\
R(\eta)=-g \eta(0)+(A-\delta) \eta(0)+(\lambda g-r \lambda) e^{-g \tau} \eta(-\tau) \\
\quad-\gamma\left[\eta(0)-\lambda e^{-g \tau} \eta(-\tau)+(\delta+\xi-A) \int_{-\tau}^{0} e^{-\xi s} e^{g s} \eta(s) \mathrm{d} s\right]
\end{array}\right.
$$

Proposition 4.7. Assume that hypotheses of Theorem 4.4 are satisfied. Then we have that

$$
\lim _{t \rightarrow+\infty} e^{-g t} K^{*}(t)=K_{L}
$$

where

$$
K_{L}=\frac{K_{I}(0)-\lambda K_{I}(-\tau)+R\left(s \mapsto \int_{s}^{0} K_{I}(u) \mathrm{d} u\right)}{1-\lambda e^{-g \tau}+R(r \mapsto-r)}
$$

Proof. Let us introduce the detrended variable $\hat{K}(t):=K(t) e^{-g t}$, since $K(t)$ satisfies (26) then $q(t)$ satisfies

$$
\begin{aligned}
& \dot{\hat{K}}(t)-\lambda e^{-g \tau} \dot{\hat{K}}(t-\tau)=-g \hat{K}(t)+(A-\delta) \hat{K}(t)+(\lambda g-r \lambda) e^{-g \tau} \hat{K}(t-\tau) \\
& -\gamma\left[\hat{K}(t)-\lambda e^{-g \tau} \hat{K}(t-\tau)+(\delta+\xi-A) \int_{-\tau}^{0} e^{-\xi s} e^{g s} \hat{K}(s+t) \mathrm{d} s\right]
\end{aligned}
$$

So using the notation $\hat{K}_{t}$, defined as:

$$
\left\{\begin{array}{l}
\hat{K}_{t}:[-\tau, 0] \rightarrow \mathbb{R} \\
\hat{K}_{t}(s):=\hat{K}(t+s),
\end{array}\right.
$$

we can rewrite the NDE solved by $\hat{K}(t)$ as

$$
\dot{\hat{K}}(t)-\lambda e^{-g \tau} \dot{\hat{K}}(t-\tau)=R\left(\hat{K}_{t}\right)
$$


whose characteristic equation is

$$
\begin{aligned}
z\left(1-\lambda e^{-g \tau} e^{-g z}\right) & =(-g+A-\delta)+\lambda(g-r) e^{-g \tau} e^{-z \tau} \\
& -\gamma\left[\left(1-\lambda e^{-g \tau} e^{-z \tau}+\frac{\delta+\xi-A}{-\xi+g+z}\left(1-e^{-(g+z-\xi) \tau}\right)\right]\right.
\end{aligned}
$$

It is easy to prove (by direct computations) that $z=0$ is a root of such a characteristic equation. Moreover it satisfies condition (1.4) in Philos and Purnaras (2004) and then, thanks to Theorem 2.1 in Philos and Purnaras (2004) one has that

$$
\hat{K}(t) \stackrel{t \rightarrow \infty}{\longrightarrow} K_{L}
$$

where

$$
K_{L}:=\frac{K_{I}(0)-\lambda K_{I}(-\tau)+R\left(s \mapsto \int_{s}^{0} K_{I}(u) \mathrm{d} u\right)}{1-\lambda e^{-g \tau}+R(r \mapsto-r)} .
$$

and this concludes the proof.

\section{Proofs}

We split this section in two parts, the first one devoted to dig deeper in the general formalism and work needed to bring the original problem to an abstract space where it is transformed into the optimal control of an ODE, and a second part proving Theorem 4.4 and Proposition 4.5 stated above.

\subsection{General formalism and approach}

We want to rewrite the problem in an equivalent form using a different formalism. More precisely we want to rewrite the evolution of the system as an evolution differential equation in an Hilbert space and use there the tools of the dynamic programming. Before using that approach we need to recall some known results. First of all we need to give a meaning to the state equation for a more general initial datum. Take $x_{0} \in \mathbb{R}, x_{1} \in L^{2}(-\tau, 0)$ and consider the neutral differential equation

$$
\left\{\begin{array}{l}
\dot{K}(t)=\lambda \dot{K}(t-\tau)+(A-\delta) K(t)-r \lambda K(t-\tau)-C(t) \\
K(0)-\lambda K(-\tau)=x_{0} \\
K(s)=x_{1}(s) \quad s \in[-\tau, 0] .
\end{array}\right.
$$


We say (following Kappel and Zhang (1986) Definition 2.1 page 118) that $K:[0,+\infty) \rightarrow \mathbb{R}$ is a generalized solution of $(36)$ if $^{7}$

1. $K \in L_{l o c}^{2}(-\tau,+\infty)$

2. $K(s)=x_{1}(s)$ a.s. on $(-\tau, 0)$

3. For almost all $t \geq 0$ one has

$$
\begin{aligned}
K(t)-\lambda K(t-\tau)= & x_{0}+\int_{-\tau}^{0}\left(\frac{r \lambda+A-\delta}{\tau} s+(A-\delta)\right)\left(K(t+s)-x_{1}(s)\right) \mathrm{d} s \\
& +\int_{0}^{t} \int_{-\tau}^{0} \frac{r \lambda+A-\delta}{\tau} K(r+s) \mathrm{d} r \mathrm{~d} s-\int_{0}^{t} C(s) \mathrm{d} s .
\end{aligned}
$$

Observe that such a definition reduces to that given in (15) when $x_{1} \in$ $C([-\tau, 0])$ and $x_{0}=x_{1}(0)-\lambda x_{1}(-\tau)$.

\subsubsection{Homogeneous case}

We consider the Hilbert space $M^{2}:=\mathbb{R} \times L^{2}(-T, 0)$ (with the scalar product $\left.\left\langle\left(x_{0}, x_{1}\right),\left(z_{0}, z_{1}\right)\right\rangle_{M^{2}}:=x_{0} z_{0}+\left\langle x_{1}, z_{1}\right\rangle_{L^{2}}\right)$. It can be proved (see Burns, Herdman and Stech (1983) Theorem 2.3 page 102) that the operator

$$
\left\{\begin{array}{l}
D(G):=\left\{\left(x_{0}, x_{1}\right) \in M^{2}: x_{1} \in W^{1,2}(-\tau, 0), x_{0}=x_{1}(0)-\lambda x_{1}(-\tau)\right\} \\
G\left(x_{0}, x_{1}\right):=\left((A-\delta) x_{1}(0)-r \lambda x_{1}(-\tau), \partial x_{1}\right)
\end{array}\right.
$$

( $\partial x_{1}$ is the derivative of the function $x_{1}$ ) is the generator of a $C_{0}$ semigroup $^{8}$ $e^{t A}$ on $M^{2}$. Kappel and Zhang (1986) in Theorem 2.2 prove that such a fact

${ }^{7}$ Note that we can rewrite the equation in the form used in Kappel and Zhang (1986) Definition 2.1 page 118 taking $G(s):=\frac{r \lambda+A-\delta}{\tau} s+(A-\delta)$ and $F(s) \equiv \frac{r \lambda+A-\delta}{\tau}$ (where $G$ and $F$ refer to the notation in Kappel and Z Zhang (1986)) indeed, for $K \in W^{\tau} 1,2(-\tau, 0)$ one has (integration by part)

$$
(A-\delta) K(0)-r \lambda K(-\tau)=\int_{-\tau}^{0}\left(\frac{r \lambda+A-\delta}{\tau} s+A-\delta\right) \dot{K}(s) \mathrm{d} s+\int_{-\tau}^{0} \frac{r \lambda+A-\delta}{\tau} K(s) \mathrm{d} s .
$$

${ }^{8}$ Actually, in our specific case, it is a $C_{0}$-group (see Burns, Herdman and Stech (1983) Theorem 2.4 page 108). 
is equivalent to the existence and uniqueness, for all choice of $\left(x_{0}, x_{1}\right) \in M^{2}$, of a generalized solution for the equation

$$
\left\{\begin{array}{l}
\dot{K}(t)=\lambda \dot{K}(t-\tau)+(A-\delta) h(t)-r \lambda K(t-\tau) \\
K(0)-\lambda K(-\tau)=x_{0} \\
K(s)=K_{1}(s) \quad s \in[-\tau, 0] .
\end{array}\right.
$$

Call such a generalized solution $\phi^{x_{0}, x_{1}}:[-\tau,+\infty)$. For $t \geq 0$ denote by $\phi_{t}^{x_{0}, x_{1}}$ the function

$$
\left\{\begin{array}{l}
\phi_{t}^{x_{0}, x_{1}}:[-\tau, 0] \rightarrow \mathbb{R} \\
\phi_{t}^{x_{0}, x_{1}}(s):=\phi^{x_{0}, x_{1}}(t+s)
\end{array}\right.
$$

The semigroup $e^{t A}$ acts as follows (Kappel and Zhang (1986) Theorem 2.2):

$$
e^{t A}\left(x_{0}, x_{1}\right):=\left(y^{x_{0}, x_{1}}(t), \phi_{t}^{x_{0}, x_{1}}\right)
$$

where

$$
\begin{array}{r}
y^{x_{0}, x_{1}}(t):=x_{0}+\int_{-\tau}^{0}\left(\frac{r \lambda+A-\delta}{\tau} s+(A-\delta)\right)\left(\phi^{x_{0}, x_{1}}(t+s)-x_{1}(s)\right) \mathrm{d} s \\
+\int_{0}^{t} \int_{-\tau}^{0} \frac{r \lambda+A-\delta}{\tau} \phi^{x_{0}, x_{1}}(r+s) \mathrm{d} r \mathrm{~d} s .
\end{array}
$$

\subsubsection{Controlled case}

Now consider $C(\cdot) \in L_{\text {loc }}^{2}(0,+\infty)$. There exists (see Burns, Herdman and Stech (1983) page 109 [where Lemma 2.6 is used]) a unique generalized solution $\phi^{x_{0}, x_{1}, C}(\cdot)$ of $(36)$. If $\left(x_{0}, x_{1}\right) \in D(G)$ (observe that this is the case in (14)) then $\phi^{x_{0}, x_{1}, C}(\cdot) \in W_{l o c}^{1,2}(0,+\infty)$ (see Burns, Herdman and Stech (1983) Theorem 2.2 page 101). Choose $\left(x_{0}, x_{1}\right) \in M^{2}$ and consider the following evolution equation in $M^{2}$ :

$$
\left\{\begin{array}{l}
\dot{x}(t)=G x(t)-(1,0) C(t) \\
x(0)=\left(x_{0}, x_{1}\right)
\end{array}\right.
$$

We say that $x \in C\left([0,+\infty) ; M^{2}\right)$ is a weak solution of (42) if, for every $\psi \in D\left(G^{*}\right)$, the function $\left\langle x(\cdot), G^{*} \psi\right\rangle$ belongs to $W_{l o c}^{1,2}(0,+\infty)$ and

$$
\left\{\begin{array}{l}
\frac{\mathrm{d}}{\mathrm{d} t}\langle x(t), \psi\rangle=\left\langle x(t), G^{*} \psi\right\rangle-C(t)\langle(1,0), \psi\rangle \\
\langle x(0), \psi\rangle=\left\langle\left(x_{0}, x_{1}\right), \psi\right\rangle .
\end{array}\right.
$$


It can be proved (see Bensoussan et al. (2007) Proposition 3.2 page 131) that (42) admits a unique weak solution that can be expressed in the following form

$$
x(t):=e^{t G}\left(x_{0}, x_{1}\right)-\int_{0}^{t} e^{(t-s) G}(1,0) C(s) \mathrm{d} s .
$$

When we use this last expression we will refer to $x(\cdot)$ as mild solution of $(42) .^{9}$

We have the following important theorem.

Theorem 5.1. The unique mild/weak solution $x(\cdot)$ of (42) is, for all $t \geq 0$ equal to:

$$
x(t)=\left(y^{x_{0}, x_{1}, C}(t), \phi_{t}^{x_{0}, x_{1}, C}\right) .
$$

Proof. See Burns, Herdman and Stech (1983), Theorem 3.1 page 110.

Denote by

$$
\begin{aligned}
y^{x_{0}, x_{1}, C}(t):= & x_{0}+\int_{-\tau}^{0}\left(\frac{r \lambda+A-\delta}{\tau} s+(A-\delta)\right)\left(\phi^{x_{0}, x_{1}, C}(t+s)-x_{1}(s)\right) \mathrm{d} s \\
& +\int_{0}^{t} \int_{-\tau}^{0} \frac{r \lambda+A-\delta}{\tau} \phi^{x_{0}, x_{1}, C}(r+s) \mathrm{d} r \mathrm{~d} s-\int_{0}^{t} C(s) \mathrm{d} s .
\end{aligned}
$$

This has the following important implications for our specific optimal control problem.

Proposition 5.2. The optimal control problem in NDE form, characterized by

(i) State equation (14) with initial datum $K_{I} \in C([-\tau, 0])$

(ii) Functional to be maximized (21)

(iii) Set of admissible controls $\mathcal{U}$ defined in (22

is equivalent to the optimal control problem in the $M^{2}$ setting characterized by

${ }^{9}$ As we have already observed in our case the notions of mild and weak solution are equivalent. In the following we will use both depending on the aspect we want to emphasize. 
(i) State equation (42) with initial datum $x_{1}:=K_{I}$ and $x_{0}:=K_{I}(0)-$ $\lambda K_{I}(-\tau)$

(ii) Functional to be maximized

$$
\int_{0}^{+\infty} e^{-\rho t} \frac{C^{1-\sigma}(t)}{1-\sigma} \mathrm{d} t
$$

(it has the same form than before)

(iii) Set of admissible controls $\mathcal{U}_{M^{2}}$ defined as

$$
\begin{aligned}
\mathcal{U}:=\left\{C:[0,+\infty): C \in L_{l o c}^{2}(0,+\infty), c \geq 0,\right. \\
\left.\quad \phi_{t}^{x_{0}, x_{1}, C}(s) \geq 0 \text { for a.e. } s \in[-\tau, 0], \text { for every } t \geq 0\right\} .
\end{aligned}
$$

Proof. It is a Corollary of Theorem 5.1.

Proposition 5.3. The adjoint of $G$ is given by

$$
\left\{\begin{aligned}
\begin{array}{rl}
D\left(G^{*}\right)=\left\{\left(y_{0}, y_{1}\right) \in M^{2}:\right. & y_{1} \in W^{1,2}(-\tau, 0) \text { and } \\
& \left.(A-\delta-r) \lambda y_{0}+\lambda y_{1}(0)-y_{1}(-\tau)=0\right\} \\
G^{*}\left(y_{0}, y_{1}\right)=\left((A-\delta) y_{0}+y_{1}(0),-\partial y_{1}\right)
\end{array}
\end{aligned}\right.
$$

Proof. $\mathbb{R} \times C^{\infty}([-\tau, 0])$ is a dense subset of $M^{2}$. For $x \in D(G)$ and $y \in$ $\mathbb{R} \times C^{\infty}([-\tau, 0])$ one has, integrating by part and then using the definition of $D(G)$

$$
\begin{gathered}
\langle G x, y\rangle=\left((A-\delta) x_{1}(0)-r \lambda x_{1}(-\tau)\right) y_{0}+\int_{-\tau}^{0}\left(\partial x_{1}(s)\right) y_{1}(s) \mathrm{d} s \\
=\left((A-\delta) x_{1}(0)-r \lambda x_{1}(-\tau)\right) y_{0}+x_{1}(0) y_{1}(0)-x_{1}(-\tau) y_{1}(-\tau) \\
-\int_{-\tau}^{0} x_{1}(s)\left(\partial\left(y_{1}(s)\right) \mathrm{d} s\right. \\
=x_{0}\left[(A-\delta) y_{0}+y_{1}(0)\right]+x_{1}(-\tau)\left[\lambda(A-\delta-r) y_{0}+\lambda y_{1}(0)-y_{1}(-\tau)\right] \\
-\int_{-\tau}^{0} x_{1}(s)\left(\partial\left(y_{1}(s)\right) \mathrm{d} s\right.
\end{gathered}
$$


so the functional $x \mapsto\langle G x, y\rangle$ can be extended (from $D(G)$ ) to the whole $M^{2}$ only if $\lambda(A-\delta-r) y_{0}+\lambda y_{1}(0)-y_{1}(-\tau)=0$ and on these elements one has $G^{*}\left(y_{0}, y_{1}\right)=\left((A-\delta) y_{0}+y_{1}(0),-\partial y_{1}\right)$, so (observe that the elements of $y \in \mathbb{R} \times C^{\infty}([-\tau, 0])$ satisfying such a condition are again a dense subset of $\left.M^{2}\right), D\left(G^{*}\right)$ is the closure of

$$
\left\{\left(y_{0}, y_{1}\right) \in \mathbb{R} \times C^{\infty}([-\tau, 0]):(A-\delta-r) \lambda y_{0}+\lambda y_{1}(0)-y_{1}(-\tau)=0\right\}
$$

w.r.t. the norm $\left|\left(y_{0}, y_{1}\right)\right|_{D\left(G^{*}\right)}:=\sqrt{\left|\left(y_{0}, y_{1}\right)\right|_{M^{2}}^{2}+\left|G^{*}\left(y_{0}, y_{1}\right)\right|_{M^{2}}^{2}}$ i.e.

$$
\begin{aligned}
D\left(G^{*}\right)=\left\{\left(y_{0}, y_{1}\right) \in M^{2}:\right. & y_{1} \in W^{1,2}(-\tau, 0) \text { and } \\
& \left.(A-\delta-r) \lambda y_{0}+\lambda y_{1}(0)-y_{1}(-\tau)=0\right\}
\end{aligned}
$$

and this concludes the proof.

The Hamilton-Jacobi-Bellman equation related to the optimal control problem (42)-(45) is

$$
\rho v(x)=\left\langle x, G^{*} D v\right\rangle_{M^{2}}+\sup _{C \geq 0}\left(-C\langle(1,0), D v(x)\rangle_{M^{2}}+\frac{C^{1-\sigma}}{1-\sigma}\right) .
$$

that, when $\langle(1,0), D v(x)\rangle_{M^{2}}>0$, reduces to

$$
\rho v(x)-\left\langle x, G^{*} D v\right\rangle_{M^{2}}-\frac{\sigma}{1-\sigma}\langle(1,0), D v(x)\rangle_{M^{2}}^{1-1 / \sigma}=0 .
$$

Definition 5.4. Given an open set $\Omega \in M^{2}$ we say that $v \in C^{1}(\Omega)$ is a solution of (49) (on $\Omega$ ) if $D v \in C\left(\Omega ; D\left(G^{*}\right)\right)$ and (50) is satisfied pointwise in all the points of $\Omega$.

Call

$$
E:=\left\{\left(x_{0}, x_{1}\right) \in M^{2}:\left(x_{0}+\beta \int_{-\tau}^{0} e^{-\xi s} x_{1}(s) \mathrm{d} s\right)>0\right\} .
$$

The next theorem is the main statement of this section, it gives a closedform solution to the HJB equation above, which will crucially serve in our application. The related (optimal) feedback is given by equation (60) here below. 
Theorem 5.5. Assume that (17), (18) and (23) hold. Denote by $P$ the elements of $M^{2}$ given by

$$
P:=\left(1, s \mapsto \beta e^{-s \xi}\right)
$$

Then a solution of (49) on $E$ is given by

$$
v\left(x_{0}, x_{1}\right)=\alpha\langle x, P\rangle^{1-\sigma}:=\alpha\left(x_{0}+\beta \int_{-\tau}^{0} e^{-\xi s} x_{1}(s) \mathrm{d} s\right)^{1-\sigma}
$$

where

$$
\alpha=\frac{1}{1-\sigma}\left(\frac{\rho-(1-\sigma) \xi}{\sigma}\right)^{-\sigma}
$$

and

$$
\beta=-\frac{\lambda(A-\delta-r)}{\lambda-e^{\xi \tau}} .
$$

Proof. We proceed with a direct computation.

Observe first that

$$
P \in D\left(G^{*}\right)
$$

indeed $P$ is in $D\left(G^{*}\right)$ if and only if

$$
\lambda(A-\delta-r)+\lambda \beta-\beta e^{\beta \tau}=0
$$

and this is true thanks to (54).

We have that

$$
D v\left(x_{0}, x_{1}\right)=\alpha(1-\sigma)\langle x, P\rangle^{-\sigma}\left(1, s \mapsto \beta e^{-\xi s}\right) .
$$

that belongs to $D\left(G^{*}\right)$ thanks to $(55$.

Observe that, using that $\xi$ satisfies (16) one has that

$$
(A-\delta)+\beta=\xi \text {. }
$$

We have that

$$
\left\langle D v\left(x_{0}, x_{1}\right),(1,0)\right\rangle=\alpha(1-\sigma)\langle x, P\rangle^{-\sigma},
$$

and (since $D v\left(x_{0}, x_{1}\right) \in D\left(G^{*}\right)$ we can apply $G^{*}$ )

$$
G^{*} D v\left(x_{0}, x_{1}\right)=\alpha(1-\sigma)\langle x, P\rangle^{-\sigma}\left((A-\delta)+\beta, s \mapsto \xi \beta e^{-\xi s}\right)
$$


so, since $\left\langle D v\left(x_{0}, x_{1}\right),(1,0)\right\rangle=\alpha(1-\sigma)\langle x, P\rangle^{-\sigma}>0$ on $E$ (note that $\alpha(1-$ $\sigma)>0$ thanks to $(23))$ we can use the expressions in (50) and check directly if our candidate solution satisfies the HJB:

$$
\begin{aligned}
\rho \alpha\langle x, P\rangle^{1-\sigma}-\alpha(1-\sigma)\langle x, P\rangle^{-\sigma} & \left\langle\left(x_{0}, x_{1}\right),\left((A-\delta)+\beta, s \mapsto \xi \beta e^{-\xi s}\right)\right\rangle \\
& -\frac{\sigma}{1-\sigma}(\alpha(1-\sigma))^{1-1 / \sigma}\langle x, P\rangle^{1-\sigma}=0
\end{aligned}
$$

that is verified if and only if

$$
\begin{aligned}
\rho\langle x, P\rangle-(1-\sigma)\left\langle\left(x_{0}, x_{1}\right),((A-\delta)+\beta, s\right. & \left.\left.\mapsto \xi \beta e^{-\xi s}\right)\right\rangle \\
& -\sigma(\alpha(1-\sigma))^{-1 / \sigma}\langle x, P\rangle=0 .
\end{aligned}
$$

Thanks to $(56)\left((A-\delta)+\beta, s \mapsto \xi \beta e^{-\xi s}\right)=\xi\left(1, s \mapsto \beta e^{-\xi s}\right)$ and then last equation becomes

$$
\rho\langle x, P\rangle-(1-\sigma) \xi\langle x, P\rangle-\sigma(\alpha(1-\sigma))^{-1 / \sigma}\langle x, P\rangle=0
$$

that is satisfied if

$$
\rho-(1-\sigma) \xi-\sigma(\alpha(1-\sigma))^{-1 / \sigma}=0
$$

that is satisfied thanks to the definition of $\alpha$ given in (53). This concludes the proof.

The feedback induced by $v$, the solution of the HJB equation found in (52) is given by

$$
\left\{\begin{aligned}
\phi: E \rightarrow \mathbb{R} & \\
\phi(x):= & \arg \max _{C \geq 0}\left(-C\langle(1,0), D v(x)\rangle_{M^{2}}+\frac{C^{1-\sigma}}{1-\sigma}\right) \\
& =\langle D v(x),(1,0)\rangle^{-1 / \sigma}=\left(\frac{\rho-(1-\sigma) \xi}{\sigma}\right)\langle x, P\rangle
\end{aligned}\right.
$$

For every initial datum $x_{0} \in E$ the equation

$$
\left\{\begin{array}{l}
\dot{x}(t)=G x(t)-(1,0) \phi(x)(t)=G x(t)-(1,0)\left(\frac{\rho-(1-\sigma) \xi}{\sigma}\right)\langle x(s), P\rangle \\
x(0)=\left(x_{0}, x_{1}\right)
\end{array}\right.
$$

(thanks to the fact that the term $(1,0)\left(\frac{\rho-(1-\sigma) \xi}{\sigma}\right)\langle x(s), P\rangle$ is Lipschitz continuous in $x$ ) has a unique mild solution $x^{*}(\cdot)$ in $C\left([0,+\infty) ; M^{2}\right)$ (see for 
example Bensoussan et al., 2007) i.e. a unique solution satisfying the integral equation

$$
x(t):=e^{t G}(x(0))-\int_{0}^{t} e^{(t-s) G}(1,0)\left(\frac{\rho-(1-\sigma) \xi}{\sigma}\right)\langle x(s), P\rangle \mathrm{d} s .
$$

Here again, as before, the notion is equivalent (see again Bensoussan et al (2007), Proposition 3.2 page 131) to that of weak solution defined in a fashion similar to (43) (one has only to change in the expression $C(t)$ with $(1,0)\left(\frac{\rho-(1-\sigma) \xi}{\sigma}\right)\langle x(t), P\rangle$

Proposition 5.6. Assume that (17), (18) and (23) hold. Provided that the related control is admissible, the feedback (60) is optimal. So the unique optimal trajectory is the unique mild/weak solution $x^{*}$ of (61) and the optimal control is given by $c^{*}(t)=\phi\left(x^{*}(t)\right)$. Moreover $v$ defined in (52) is the value function of the problem.

Proof. It can be proved using the same arguments used for example in Boucekkine, Camacho and Fabbri (2010) Theorem 3.1.

\subsection{Application to our problem}

With the material developed in the previous sub-section, the main outcomes of the dynamic programming method stated in Section 4 can be almost directly proved, particularly Theorem 4.4 giving the closed-form value function.

Proof of Theorem 4.4. The statements of the theorem comes from those of Theorem 5.5 and Proposition 5.6 once we rewrite them in the NDE formalism (using the equivalence of the two formalisms stated in Proposition 5.2).

The constancy of optimal detrended consumption requires a little bit more work.

Proof of Proposition 4.5. We use again the $M^{2}$-setting to prove the result. Since the optimal trajectory satisfies (61) we have (using the definition of weak solution), that, taking the scalar product with $P$, defined in (51) (observe that we already checked in (55) that $P \in D\left(G^{*}\right)$ ). 
We have

$$
\begin{aligned}
\frac{\mathrm{d}\langle x(t), P\rangle}{\mathrm{d} t} & =\left\langle x(t), G^{*} P\right\rangle-\langle(1,0), P\rangle \phi(x(t)) \\
=\langle x(t),((A-\delta)+\beta, s & \left.\left.\mapsto \xi \beta e^{-\xi s}\right)\right\rangle-\left(\frac{\rho-(1-\sigma) \xi}{\sigma}\right) \\
& =\left[\xi-\left(\frac{\rho-(1-\sigma) \xi}{\sigma}\right)\right]\langle x(t), P\rangle
\end{aligned}
$$

So

$$
\langle x(t), P\rangle=\langle x(0), P\rangle e^{\left[\xi-\left(\frac{\rho-(1-\sigma) \xi}{\sigma}\right)\right] t}=\langle x(0), P\rangle e^{\left[\frac{\xi-\rho}{\sigma}\right] t} .
$$

So, once one define $g:=\frac{\xi-\rho}{\sigma}$,

$$
C^{*}(t)=\phi(x(t))=\frac{\rho-(1-\sigma) \xi}{\sigma}\langle x(t), P\rangle=\frac{\rho-(1-\sigma) \xi}{\sigma}\langle x(0), P\rangle e^{g t}
$$

and we have the expression of $C^{*}(t)$ given in the claim of Proposition 4.5 once we translate expression above in the NDE setup.

Remark 5.7. The co-state variable in the infinite dimensional context is

$$
\begin{aligned}
e^{-\rho t} D v\left(x_{0}(t), x_{1}(t)\right) & =e^{-\rho t} \alpha(1-\sigma)\langle x(t), P\rangle^{-\sigma}\left(1, s \mapsto \beta e^{-\xi s}\right) \\
& =e^{-\rho t}\left(\frac{\rho-(1-\sigma) \xi}{\sigma}\right)^{-\sigma} e^{-g \sigma t}\left(1, s \mapsto \beta e^{-\xi s}\right) .
\end{aligned}
$$

The co-state of the finite dimensional setting $q(t)$, introduced in Proposition 3.2 , corresponds to its first component i.e. to $e^{-\rho t} D_{x_{0}} v\left(x_{0}(t), x_{1}(t)\right)\left(e^{-\rho t}\right.$ times the partial derivative of $v$ w.r.t. the first (one-dimensional) component. We have

$$
\begin{aligned}
e^{-\rho t} D_{x_{0}} v\left(x_{0}(t), x_{1}(t)\right)=\alpha(1-\sigma)\langle x(t), P\rangle^{-\sigma} & = \\
e^{-\rho t}\left(\frac{\rho-(1-\sigma) \xi}{\sigma}\right)^{-\sigma} e^{-g \sigma t} & =\left(\frac{\rho-(1-\sigma) \xi}{\sigma}\right)^{-\sigma} e^{-\xi t}
\end{aligned}
$$

It immediate to observe that it satisfies (6) (verifying it directly using the expression of $C(t)$ given in Proposition 4.5). Moreover, since $\xi$ satisfies (16), we can easily see that the expression in (64) satisfies (7) too. 


\section{References}

P. Asea and P. Zak. Time-to-build and cycles. Journal of Economic Dynamics and Control 23, 1155-1175 (1999).

A. Bensoussan, G. Da Prato, M. C. Delfour and S. K. Mitter. Representation and control of infinite dimensional system. Second edition. Birkhäuser, Boston, 2007.

M. Bambi and O. Licandro. Endogenous growth and wave-like business fluctuations. Mimeo, 2011.

M. Bambi, G. Fabbri and F. Gozzi. Optimal policy and consumption smoothing effects in the time-to-build AK model. Mimeo, 2010. Forthcoming in Economic Theory.

M. Bambi. Endogenous growth and time to build: the AK case. Journal of Economic Dynamics and Control 32, 1015-1040 (2008).

R. Boucekkine and P. Pintus. Is History a Blessing or a Curse? International Borrowing without Commitment, Leapfrogging and Growth Reversals. Discussion Paper IRES 2010-38, Université catholique de Louvain, 2010 .

R. Boucekkine, C. Camacho and G. Fabbri. Spatial dynamics and convergence: the spatial AK model. Working Papers 2010-06, Department of Economics, University of Glasgow, 2010.

R. Boucekkine, G. Fabbri and F. Gozzi. Maintenance and investment: complements or substitutes? A reappraisal. Journal of Economic Dynamics and Control 34, 2420-2439 (2010).

R. Boucekkine, O. Licandro, L. Puch and F. del Río. Vintage capital and the dynamics of the AK model. Journal of Economic Theory 120, 39-76 (2005).

R. Boucekkine, M. Germain and O. Licandro. Replacement echoes in the vintage capital growth model. Journal of Economic Theory 74, 333-348 (1997) 
A. Brumbey. On the asymptotic behaviour of solutions of differentialdifference equations of neutral type. Journal of Differential Equations 7, $175-188(1970)$

J.A. Burns, T.L. Herdman, and H.W. Stech. Linear functional differential equations as semigroups on product spaces. SIAM Journal on Mathematical Analysis, 14-98 (1983).

F. Collard, O. Licandro and L. Puch. The short run dynamics of optimal growth models with delays. Annales d'Economie et Statistique 90, 127-144 (2008)

G. Fabbri and F. Gozzi. Solving optimal growth models with vintage capital: The dynamic programming approach. Journal of Economic Theory 143, 331-373 (2008)

G. Fabbri, S. Faggian and F. Gozzi. On dynamic programming in economic models governed by DDEs. Mathematical Population Studies 15, 267-290 (2008)

G. Feichtinger, R. Hartl, P. Kort and V. Veliov. Capital accumulation under technological progress and learning: a vintage capital approach. European Journal of Operation Research 172, 293-310 (2006)

J. K. Hale and S. M. Verduyn Lunel. Introduction to functional differential equations. Springer-Verlag, Berlin, 1993.

N. Hritonenko and Y. Yatsenko. Turnpike and optimal trajectories in integral dynamic models with endogenous delay. Journal of Optimization Theory and Applications 127, 109-127 (2005)

Johansen L., Substitution Versus fixed production coefficients in the theory of economic growth, Econometrica 27, 157176 (1959).

M. Kalecki. A Macrodynamic theory of business cycles. Econometrica 3, 327344 (1935).

F. Kappel and K.P. Zhang. Equivalence of functional-differential equations of neutral type and abstract Cauchy problems. Monatshefte fur Mathematik 101, 115-133 (1986) 
V. Kolmanovski and A. Myshkis. Introduction to the theory and applications of functional differential equations. Kluwer Academic Publishers, 1999.

I. Kordonis, N. Niyianni and C. Philos. On the behaviour of the solutions of scalar first-order autonomous neutral differential equations. Arch. Math. 71, 454-464 (1998)

J. Malcomson. Replacement and the rental value of capital equipment subject to obsolescence. Journal of Economic Theory 10, 24-41 (1975)

C. Philos and I. Purnaras. Asymptotic properties, nonoscillation, and stability of scalar first-order autonomous linear neutral differential equations. Electronic Journal of Differential Equations 3, 1-17 (2004)

R. Solow, J. Tobin, C. Von Weizsacker and M. Yaari. Neoclassical growth with fixed factor proportions. Review of Economic Studies 33, 79-115 (1966)

S. Xu, J. Lam and C. Yang. Robust $H_{\infty}$ control for uncertain linear neutral delay systems. Optimal Control Applications and Methods 23, 113-123 (2002) 